\title{
Locating a metro line in a historical city centre: application to Sevilla
}

\author{
G Laporte ${ }^{1 *}$, JA Mesa ${ }^{2}$, FA Ortega ${ }^{2}$ and MA Pozo ${ }^{2}$ \\ ${ }^{1}$ HEC Montréal, Canada; and ${ }^{2}$ Universidad de Sevilla, Sevilla, Spain
}

\begin{abstract}
When constructing a metro alignment under a historical city centre, it is important to generate a cost-effective path while maintaining a minimum distance between the alignment and historical buildings. This paper describes a simple methodology for generating a set of good alternative solutions. It is based on the use of Voronoi diagrams. The method was applied to data from the city of Sevilla.
\end{abstract}

Keywords: constrained shortest path problem; metro alignment; historical buildings; Voronoi diagrams

\section{Introduction}

Metro construction projects are complex and costly endeavours involving several players such as city planners, civil and traffic engineers, geologists, politicians, environmentalists and citizen interest groups. This is an area where operational research has traditionally played a minor role (Magnanti and Wong, 1984; Gendreau et al, 1995). However, in recent years a number of analytical tools have been developed to assess alternative network configurations (Musso and Vuchic, 1988; Laporte et al, 1994; Gattuso and Miriello, 2005) or to design alignments (Bruno et al, 2001; Bruno and Laporte, 2002; Vuchic, 2004; Laporte et al, 2005). None of the available methods can claim to provide a complete and definite solution to a metro planning problem as there are just too many players, criteria and uncertainties present in such projects. However, operational research can be useful in generating and assessing alternative solutions and in solving specific subproblems.

One of the main steps of a metro construction project is the design of a network consisting of nodes and edges. The nodes correspond to stations while the edges are the links between two consecutive stations. Such networks are designed with the aim of improving population mobility while providing shorter travel times. This is usually achieved by maximizing population or trip coverage (Laporte et al, 2005), subject to a variety of constraints. For example, it is common to impose a lower and an upper limit on the length of any link (Bruno et al, 2001). These limits differ for the inner-city and the suburban sections of the alignments. For an overview of optimization methods in transportation planning, see Laporte et al (2000).

Metro maps are stylized representations of the actual network, like the famous London underground map initially

\footnotetext{
*Correspondence: G Laporte, HEC Montréal, 3000 chemin de la Côte-Sainte-Catherine, Montreal, Canada H3T 2 A7.

E-mail: gilbert@crt.umontreal.ca
}

designed by Harry Beck (Wikipedia). They typically represent edges by straight segments, or lines making $45^{\circ}$ or $90^{\circ}$ angles, but reality is often different because for a number of engineering considerations inter-station links have to follow curved or meandering paths. This is precisely the case in the problem considered in this paper. Our problem is motivated by the construction of a metro link under the historical centre of the city of Sevilla. The Sevilla metro system (see Figure 1) has been in several stages of planning, replanning and construction since the 1970s. The construction of line 1 of the initial project (La Plata-Pino Montano) had to be interrupted in 1984 after it damaged several historical and patrimonial buildings. Construction has now resumed with a different trajectory. Line 2 will connect the Sevilla west gate (Puerta Triana) to the airport and the neighbourhood of Torreblanca. It will cross the historical centre of Sevilla between the Plaza de Armas bus station and the Santa Justa railway station (Figure 2). The absence of wide streets in the historical centre prevents excavation and forces the digging of a $2.5 \mathrm{~km}$ tunnel. To complicate matters, the tunnel must stay clear of historical buildings in that area. This constraint is imposed to avoid digging too close to these buildings and thus incur the risk of weakening their foundations, namely because landslides often occur during the construction. It is also preferable to construct the metro alignments far from fragile buildings to reduce the effects of vibrations. The inclusion of this consideration in the design forces a circumvoluting pattern and considerably increases construction costs. A question of interest is how to generate such non-linear alignments without deviating too much from the most direct trajectory.

In this note we propose a simple and workable methodology for this problem. Tests conducted on the city of Sevilla data confirm the feasibility of the proposed approach. 


\section{Methodology}

The problem is defined in a planar region containing a set of nodes $V=\{1, \ldots, n\}$ representing historical buildings, as well as an origin 0 and a destination $n+1$ for the metro alignment. The aim is to construct a shortest undirected path between 0 and $n+1$ that will not be too close to the nodes of $V$. This requirement can be enforced through the definition of suitable constraints and objectives in the shortest path optimization model. In our problem, the alignment must stay clear of a safe circle of radius $r$ around each node of $V$. To better enforce a minimum separation distance between the path and a node, one can use a secondary objective function consisting of maximizing the minimum distance between the path and a node. We call this path a maxmin path. This yields a bicriteria problem (shortest length and maxmin distance), subject to a minimum separation constraint. This type of problem has applications in other areas, such as sensor exposure problems (Megerian et al, 2005) arising in telecommunications and in military contexts. For example, an agent may have to forge through a terrain in which sensors

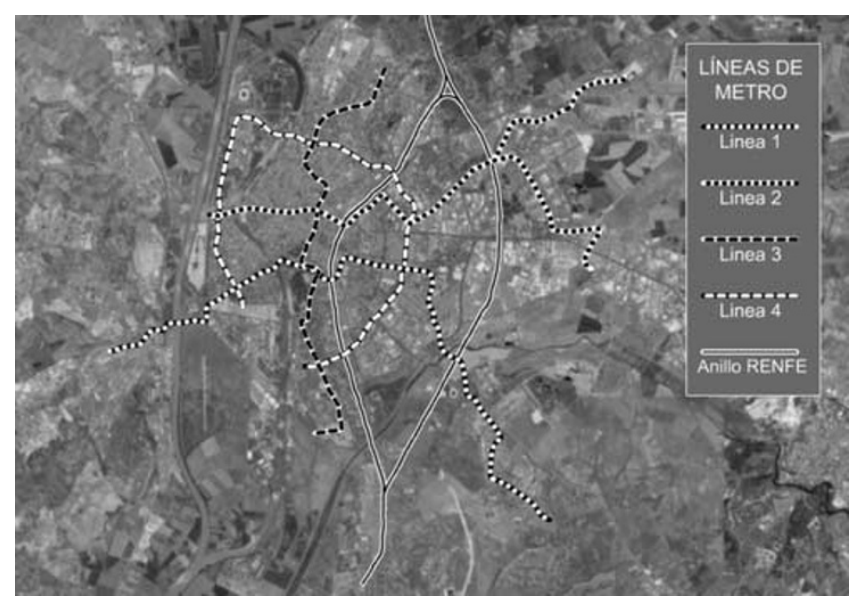

Figure 1 Metro network of Sevilla. have been located by an enemy. The aim of the agent is to determine a path while keeping at a safe distance from the sensors.

An interesting result proved by Megerian et al (2005) is the following. Assume that all nodes are enclosed in a bounded area. Consider the Voronoi diagram induced by the nodes of $V \cup\{0, n+1\}$. Then at least one maxmin path from 0 to $n+1$ uses only Voronoi edges (Figure 3). Because in our application no minimum separation distance is imposed on 0 and $n+1$, the two edges incident to these nodes do not have to coincide with edges of the Voronoi diagram. A Voronoi diagram can be constructed in $O(n \log n)$ time (see, eg, Okabe et al, 1999). Our algorithm can be described as follows:

Step 1: Read the coordinates of the nodes of $V$ together with the start node 0 and the end node $n+1$. Construct the Voronoi diagram.

Step 2: For a given safety radius $r$, extract from the Voronoi diagram a graph $G(r)=(V, E(r))$ obtained after eliminating the edges of the Voronoi diagram within a distance $r$ of a node of $V$.

Step 3: Compute a shortest path $P$ in $G(r)$ between nodes 0 and $n+1$. If no path exists, stop.

Step 4: Let $e \in E(r)$ be the edge of $P$ closest to a node of $V$. In case of ties $e$ is the longest edge. Let $E(r):=$ $E(R) \backslash\{e\}$. Go to Step 3 .

This procedure results in a set of different paths among which the decision maker can choose.

In our modelling approach we have chosen to represent buildings by nodes. However, the same methodology still works if the buildings are represented by convex polygons. In this case area Voronoi diagrams can still be constructed in $O(n \log n)$ time, as explained by Kedem et al (1986). The iterative shortest path computation remains unchanged.

Beyond its simplicity, another advantage of this method lies in the fact that it produces paths having a good separation

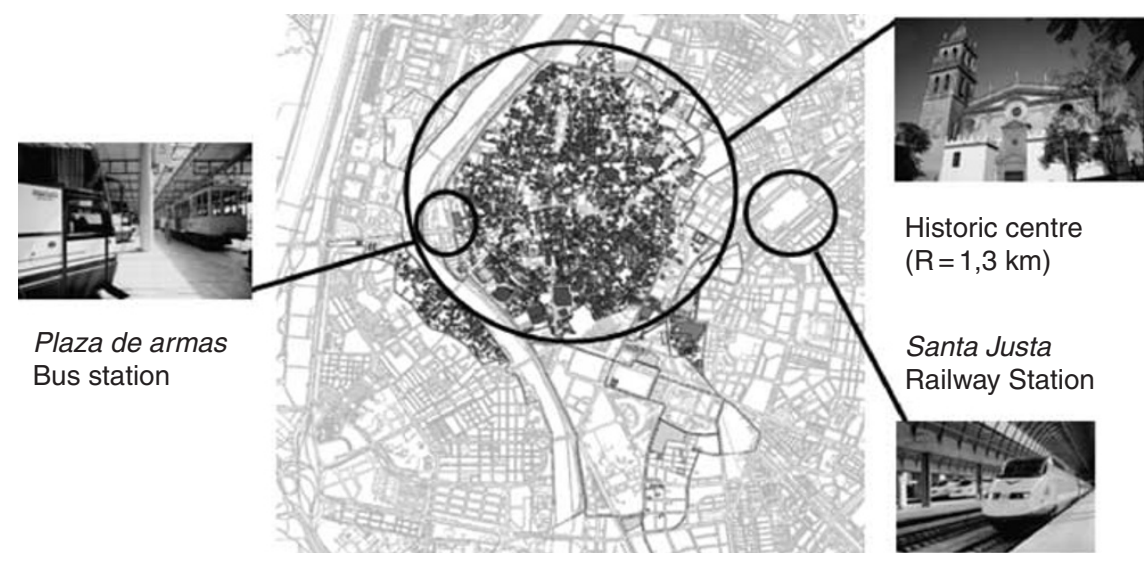

Figure 2 Historical centre of Sevilla. 

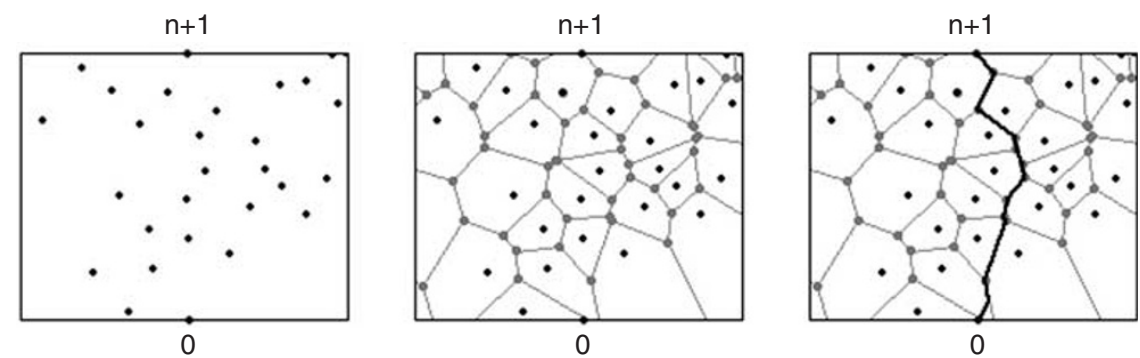

Figure 3 Illustration of the algorithm proposed by Megerian et al (2005).
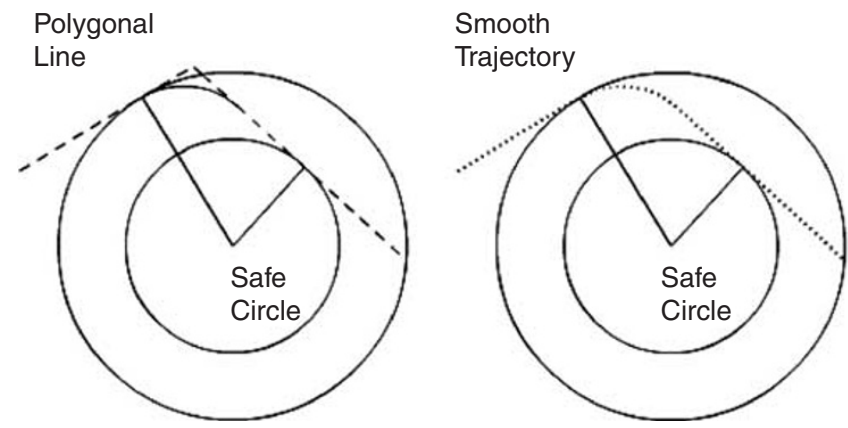

Figure 4 Smoothing a bend in a polygonal alignment.

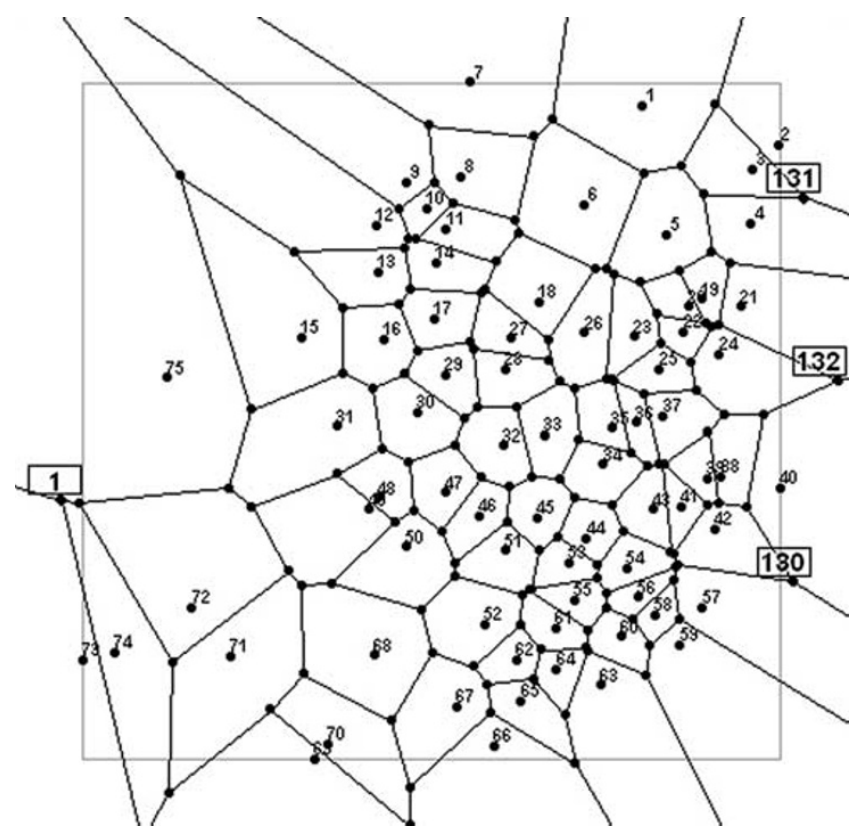

Figure 5 Voronoi diagram for the historical buildings in the centre of Sevilla.

from all nodes, not just the two associated with the critical edge. Indeed, any point on edge of the Voronoi diagram is equidistant from the two nodes located in the Voronoi areas adjacent to that edge. As a result any point on the maxmin path

maximizes the minimal separation to the two nodes closest to that point.

In practice solutions provided by a maxmin path on a Voronoi diagram may need to be smoothened, that is, angles may have to be replaced with smooth curves (Figure 4) drawn by means of cubic splines (de Boor, 1978), Hermite interpolation (Kahaner et al, 1989), or calculus-based techniques (Ortega et al, 2004), which is the method we advocate.

\section{Application to the city of Sevilla}

The methodology just described was applied to the design of several potential alignments from vertex 1 to one of nodes 130, 131 and 132 in the historical centre of Sevilla (Figure 5). Figure 5 depicts the full Voronoi diagram while Figure 6 corresponds to a simplified diagram obtained after removing the infeasible Voronoi edges. The coordinates 

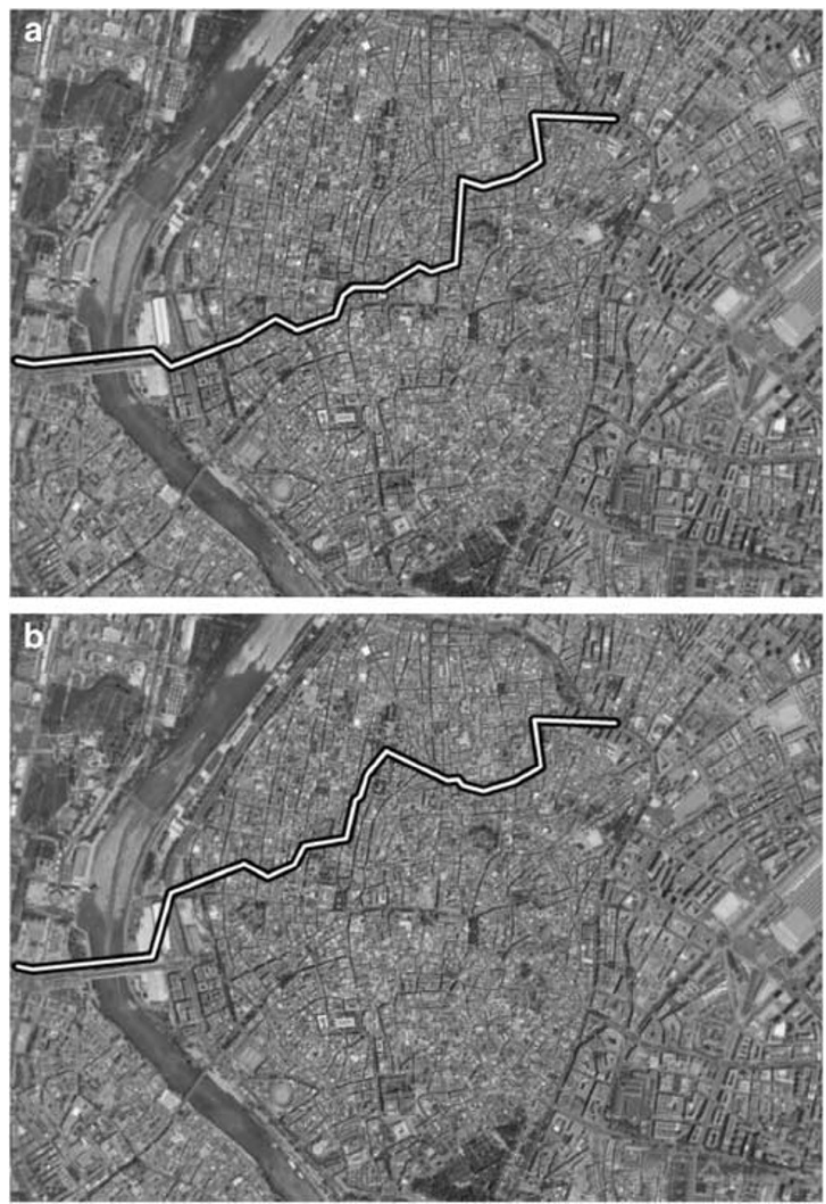

Figure 7 Two alternative feasible paths between nodes 1 and 131 shown on the Sevilla map. (a) First path; (b) Second path.

are provided in the Appendix. To illustrate, we report the computational results corresponding to two different paths between nodes 1 and 131. It can be seen that these two paths are quite different from each other. The first path has a length of $3134.8 \mathrm{~m}$ and passes $80.7 \mathrm{~m}$ away from the closest building. The second path, which is $3176.8 \mathrm{~m}$ long, passes $88.1 \mathrm{~m}$ away from the closest building. These paths are illustrated on the Sevilla map (Figure 7). Neither of these two solutions dominates the other. The final choice rests with the decision maker who can base his or her choice on the criteria used to produce the alternative solutions, but also on other considerations, some of which are difficult to quantify.

\section{Conclusion}

We have described a simple methodology for the construction of a metro alignment through a historical city centre. The aim was to produce cost-effective solutions under constraints imposing a minimum separation between the alignment and historical buildings. The proposed approach was successfully applied to data from the city of Sevilla.

Acknowledgements - This work was partially supported by the Canadian Natural Sciences and Engineering Research Council under Grant 39682-05, by the Future and Emerging Technologies Unit of EC (IST priority-6th FP), under contract no. FP6-021235-2 (project ARRIVAL), and by the Spanish Research Project 70029/T05. This support is gratefully acknowledged. Thanks are due to the referees for their valuable comments.

\section{References}

de Boor C (1978). A Practical Guide to Splines. Springer-Verlag: New York.

Bruno $G$ and Laporte $G$ (2002). An interactive decision support system for the design of rapid public transit networks. INFOR $\mathbf{4 0}$ : 111-118.

Bruno G, Gendreau M and Laporte G (2001). A heuristic for the location of a rapid transit line. Comput Opns Res 29: 1-12.

Gattuso D and Miriello E (2005). Compared analysis of metro networks supported by graph theory. Networks Spatial Econ 5: 395-414.

Gendreau M, Laporte G and Mesa JA (1995). Locating rapid transit lines. J Adv Transport 29: 145-162.

Kahaner D, Moler C and Nash S (1989). Numerical Methods and Software. Prentice-Hall: Englewood Cliffs, NJ.

Kedem K, Livne R, Pach J and Sharir M (1986). On the union of Jordan regions and collision-free translational motion amidst polygonal obstacles. Discrete Comput Geom 1: 59-71.

Laporte G, Mesa JA and Ortega FA (1994). Assessing topological configurations for rapid transit networks. Stud Locat Anal 7: $105-121$.

Laporte G, Mesa JA and Ortega FA (2000). Optimization methods for the planning of rapid transit lines. Eur $J$ Opl Res 122: $1-10$

Laporte G, Mesa JA, Ortega FA and Sevillano I (2005). Maximizing trip coverage in the location of a single rapid transit alignment. Ann Opns Res 136: 49-63.

Magnanti TL and Wong RT (1984). Network design and transportation planning: Models and algorithms. Transport Sci 18: 1-55.

Megerian S, Koushanfar F, Potkonjak M and Srivastava MB (2005). Worst and best-case coverage in sensor networks. IEEE Trans Mobile Comput 4: 84-92.

Musso A and Vuchic VR (1988). Characteristics of metro networks and methodology for their evaluation. Transport Res Rec 1162: 22-33.

Okabe A, Boots B, Sugihara K and Chin SN (1999). Spatial Tessellations: Concepts and Applications of Voronoi Diagrams, 2nd edn. Wiley: Chichester.

Ortega FA, Mesa JA and Laporte G (2004). Generación de alineamientos no zigzagueantes de máxima cobertura de viaje en el diseño de sistemas urbanos de transporte. Actas del VI Congreso de Ingeniería del Transsporte, Publicaciones Universidad de Zaragoza, Zaragoza, pp 703-710.

Vuchic V (2004). Urban Transit: Operations, Planning and Economics. Wiley: New York.

Wikipedia, http://en.wikipedia.org/wiki/Tube_map. 


\section{Appendix}

Coordinates for the nodes represented in Figures 5 and 6.

$\begin{array}{ll}\text { 1: }(2219,2408) & 26:(2035,1675) \\ \text { 2: }(2658,2280) & 27:(1795,1658) \\ \text { 3: }(2577,2204) & 28:(1780,1558) \\ \text { 4: }(2571,2028) & 29:(1584,1541) \\ \text { 5: }(2299,1990) & 30:(1494,1417) \\ \text { 6: }(2033,2090) & 31:(1237,1373) \\ \text { 7: }(1666,2483) & 32:(1772,1314) \\ \text { 8: }(1634,2180) & 33:(1906,1346) \\ \text { 9: }(1460,2162) & 34:(2096,1252) \\ \text { 10: }(1527,2076) & 35:(2124,1368) \\ 11:(1589,2011) & 36:(2199,1386) \\ \text { 12: }(1362,2023) & 37:(2289,1407) \\ \text { 13: }(1368,1872) & 38:(2476,1214) \\ \text { 14: }(1556,1903) & 39:(2432,1208) \\ \text { 15: }(1122,1661) & 40:(2666,1178) \\ 16:(1386,1656) & 41:(2346,1117) \\ 17:(1549,1720) & 42:(2453,1044) \\ 18:(1888,1773) & 43:(2255,1109) \\ 19:(2414,1783) & 44:(2041,1015) \\ \text { 20: }(2369,1760) & 45:(1880,1079) \\ \text { 21: }(2541,1759) & 46:(1692,1085) \\ \text { 22: }(2355,1678) & 47:(1587,1162) \\ 23:(2196,1664) & 48:(1367,1144) \\ 24:(2470,1607) & 49:(1338,1109) \\ 25:(2277,1558) & 50:(1458,987)\end{array}$

51: $(1781,975)$

52: $(1712,735)$

53: $(1983,935)$

54: $(2171,918)$

55: $(2004,815)$

56: $(2207,824)$

57: $(2416,790)$

58: $(2260,768)$

59: $(2343,670)$

60: $(2153,697)$

$61:(1940,723)$

62: $(1815,622)$

63: $(2087,541)$

64: $(1945,591)$

65: $(1830,489)$

66: $(1744,345)$

67: $(1623,473)$

68: $(1356,638)$

69: $(1164,303)$

70: $(1204,351)$

71: $(894,634)$

72: $(768,793)$

73: $(414,621)$

74: $(519,646)$

75: $(689,1531)$ 